\title{
Layer charge instability in unbalanced bilayer systems in the quantum Hall regime
}

\author{
E. Tutuc, R. Pillarisetty, S. Melinte, E.P. De Poortere, and M. Shayegan \\ Department of Electrical Engineering, Princeton University, Princeton, NJ 08544
}

(Dated: October 31, 2018)

\begin{abstract}
Measurements in GaAs hole bilayers with unequal layer densities reveal a pronounced magnetoresistance hysteresis at the magnetic field positions where either the majority or minority layer is at Landau level filling factor one. At a fixed field in the hysteretic regions, the resistance exhibits an unusual time dependence, consisting of random, bidirectional jumps followed by slow relaxations. These anomalies are apparently caused by instabilities in the charge distribution of the two layers.

PACS numbers: 73.50.-h, 71.70.Ej, 73.43.Qt
\end{abstract}

Hysteretic phenomena are widespread in nature. They are common magnetic materials, and often indicate a non-equilibrium situation associated with a phase transition and the presence of domains [1]. Recently, hysteresis has also been reported in various two-dimensional (2D) carrier systems in semiconductor structures at low temperatures and high magnetic fields $[2,3,4,4,5,6,7]$. In these cases, magneto-resistance $\left(\rho_{x x}\right)$ hysteresis appears in the quantum Hall $(\mathrm{QH})$ regime when two Landau levels (LLs) with opposite spin are brought into coincidence. While the 2D systems studied have been notably different, the common thread in these experiments is that there is a magnetic transition involving the carrier spin 8].

Here we present hysteretic $\rho_{x x}$ data in 2D bilayer systems in the $\mathrm{QH}$ regime. The hysteresis in these systems has a different origin and is caused by a non-equilibrium charge distribution in the two layers. We studied the magneto-transport coefficients of GaAs bilayer hole systems with unequal layer densities. When the interlayer tunneling is sufficiently small, $\rho_{x x}$ of the bilayer system exhibits a pronounced hysteresis at perpendicular magnetic field $(B)$ positions close to where either the majority or minority layer is at LL filling factor one. Most remarkable is the time dependence of $\rho_{x x}$ at a fixed field in the hysteretic regime, when the two layers are closely spaced. As a function of time, $\rho_{x x}$ exhibits large, random, sudden jumps toward higher and lower values, followed by a slow decay in the opposite direction. The data may signal an instability in the charge distribution of the two layers, i.e., an instability associated with the pseudospin (layer), rather than spin, degree of freedom.

We studied nine GaAs bilayer hole samples from six different wafers, all grown on GaAs (311)A substrates and modulation doped with Si. In all samples, the holes are confined to two $15 \mathrm{~nm}$-wide GaAs quantum wells which are separated by AlAs or AlAs/AlGaAs barriers with thickness $7.5 \leq W \leq 200 \mathrm{~nm}$. The rather thick barrier combined with the large effective mass of GaAs 2D holes [9] reduces considerably the tunneling between the two layers 10. As grown, the samples have layer densities of $\leq 7 \times 10^{10} \mathrm{~cm}^{-2}$, and low temperature $(T)$ mobilities of $\sim 35 \mathrm{~m}^{2} /$ Vs. Metallic top and bottom gates were added to control the densities in the layers. We studied several types of devices, including $2.5 \times 2.5 \mathrm{~mm}$ square samples and ones with patterned Hall bars; in these samples the ohmic contacts contact both layers. One sample was fabricated using a selective depletion scheme 11] that allows probing the transport characteristics of individual layers. The measurements were performed in a dilution refrigerator down to $T=20 \mathrm{mK}$.

Data of Fig. 1 highlight some of the results of our study. In (a) we show a set of traces where $\rho_{x x}$ was measured for a sample with $W=11 \mathrm{~nm}$ as $B$ was ramped up or down. For the traces of Fig. 1(a) the total bilayer density $\left(p_{t o t}\right)$ is kept constant at $5.5 \times 10^{10} \mathrm{~cm}^{-2}$ while charge is transferred from one layer to another using back- and front-gate biases. We define the charge transfer from one layer to another as $\Delta p=\left(p_{B}-p_{T}\right) / 2$, where $p_{B}$ and $p_{T}$ are the densities of bottom and top layers, respectively [12. At a given value of $B$, we define the filling factor, $\nu$, of the bilayer system as the ratio between $p_{t o t}$ and the LL degeneracy, $e B / h$. We also introduce the filling factors for top and bottom layers, $\nu_{T}$ and $\nu_{B}$ respectively, as the ratio between the layer density and $e B / h$.

The data of Fig. 1(a) show that when the bilayer system is balanced $\left(\Delta p=0\right.$; top trace) $\rho_{x x}$ is independent of the direction $B$ is ramped. However, as soon as the system is imbalanced $(|\Delta p|>0)$ a strong hysteresis develops in $\rho_{x x}$. For values of $\Delta p<4.7 \times 10^{9} \mathrm{~cm}^{-2}$, $\rho_{x x}$ displays hysteretic behavior in two field ranges near $\nu=2$, one near $\nu_{T}=1$ and another near $\nu_{B}=1$. When $\Delta p \geq 4.7 \times 10^{9} \mathrm{~cm}^{-2}$, the hysteresis exists only near $\nu_{B}=1$. For sufficiently large $\Delta p$, no hysteresis is observed. The amplitude of the hysteresis also decreases as $T$ is increased (data not shown) and vanishes completely above $T \simeq 230 \mathrm{mK}$, roughly independent of $\Delta p$.

To probe the contribution of the spin degree of freedom to the hysteresis in our bilayer systems, we performed measurements in tilted magnetic fields on a sample very similar to the one shown in Fig. 1(a). In this experiment the direction of the field was kept at an angle $\theta$ with respect to the normal to the plane of the bilayer system. For $\theta$ ranging from $0^{\circ}$ to $80^{\circ}$, corresponding to a six-fold increase of the total field (and therefore of the Zeeman energy) in the hysteretic region, the position of the hysteresis in perpendicular magnetic field did not change at all. If the hysteresis were caused by an instability associated with the spin degree of freedom, one would expect 6, 7] that the applied parallel field would change the po- 
(a)

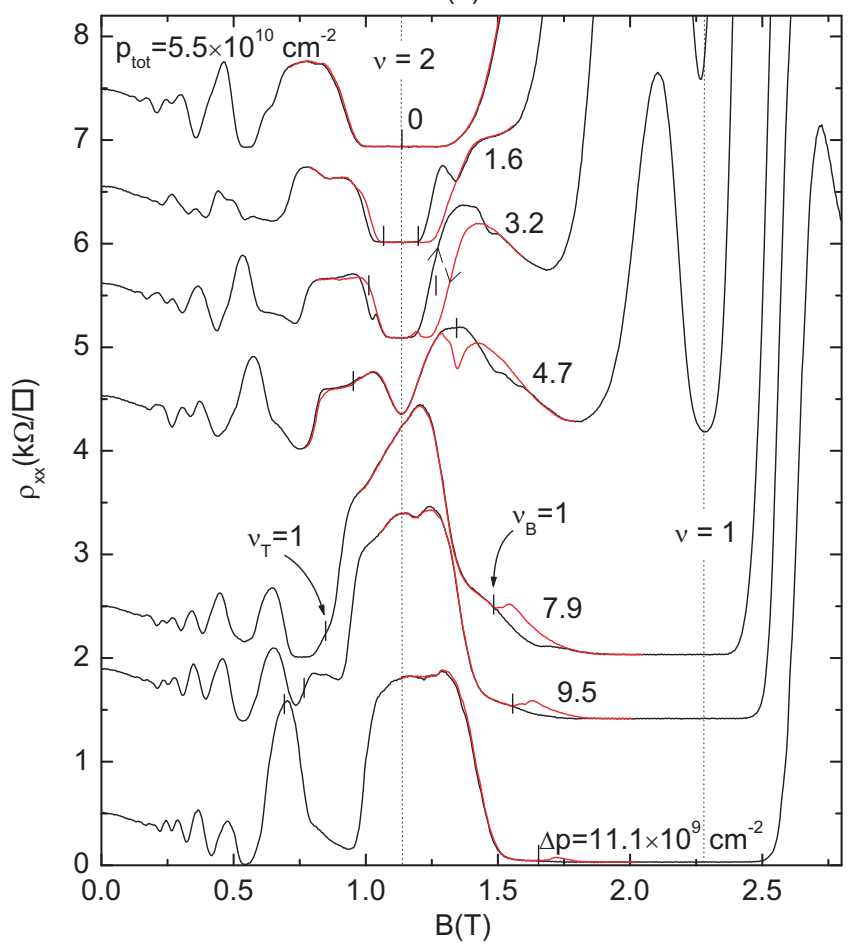

$\mathrm{p}_{\mathrm{tot}}=10.8 \times 10^{10} \mathrm{~cm}^{-2} \quad \Delta \mathrm{p}=1.3 \times 10^{10} \mathrm{~cm}^{-2}$

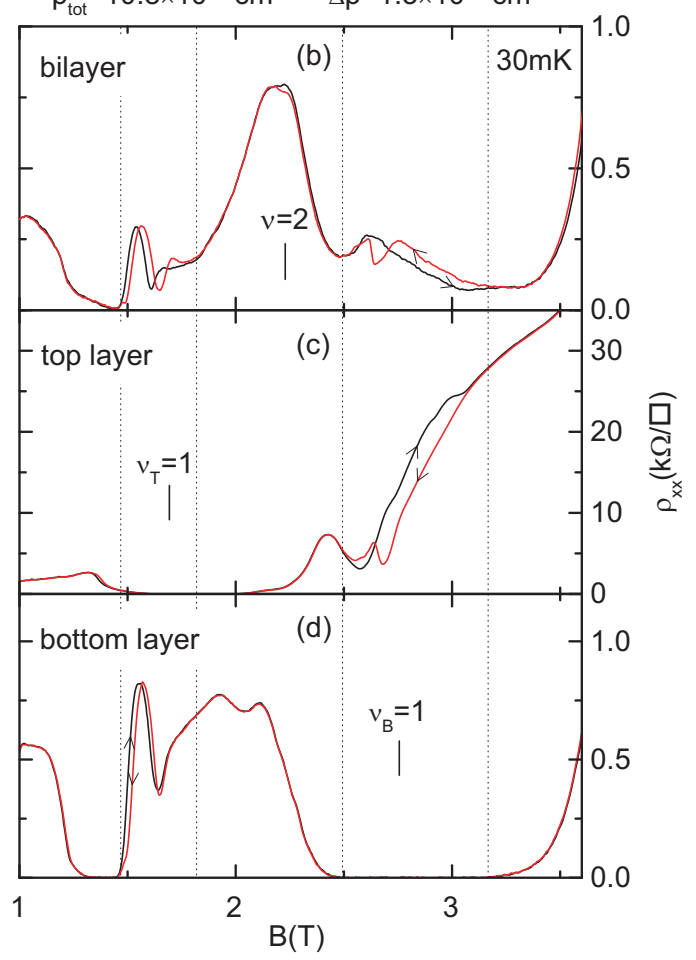

FIG. 1: (a) $\rho_{x x}$ vs. $B$ traces for a GaAs bilayer hole sample with a barrier width $W=11$ nm. Data are shown for different values of charge transfer, $\Delta p$, while $p_{\text {tot }}$ is kept constant at $5.5 \times 10^{10} \mathrm{~cm}^{-2}$. The traces are shifted vertically for clarity. The dotted lines indicate the positions of bilayer filling factors $\nu=2$ and $\nu=1$. A strong hysteresis develops in $\rho_{x x}$ when the bilayer system is imbalanced. The right and left tick marks in each trace indicate the estimated positions of filling factor one for layers with higher (bottom) and lower (top) densities, respectively. The right panel shows data for a similar sample but with independent layer contacts, and $p_{t o t}=10.8 \times 10^{10} \mathrm{~cm}^{-2}$ and $\Delta p=1.3 \times 10^{10} \mathrm{~cm}^{-2}$. (b) $\rho_{x x}$ vs. $B$ traces measured when the ohmic contacts are connected to both layers. (c) and (d) $\rho_{x x}$ of the top and bottom layers measured individually. In all figures the black (red) line represents the trace taken when $B$ is swept up (down).

sition and magnitude of the hysteresis. These results rule out spin as being responsible for the hysteresis.

To better understand the origin of the observed hysteresis, we fabricated another sample from a different wafer, also with $W=11 \mathrm{~nm}$, using a selective depletion scheme [11], and aimed to independently probe each layer of the bilayer system. The data are shown in the right panel of Fig. 1. In (b) we plot $\rho_{x x}$ for the bilayer system, that is, when the ohmic contacts are connected to both layers, for both up and down $B$-sweeps. In (c) and (d) we show $\rho_{x x}$ traces for the top and bottom layers, measured separately, but at the same pair of layer densities as in panel (b) traces. Two features of these data are noteworthy. First, the traces of (b) exhibit hysteresis in two ranges of $B$. The hysteresis between 1.45 and $1.8 \mathrm{~T}$ matches well the position of $\nu_{T}=1 \mathrm{QH}$ state of the top layer as seen in (c), while the hysteresis located between 2.5 and $3.2 \mathrm{~T}$ overlaps the $\mathrm{QH}$ state of the bottom layer (see (d)). This observation confirms that the hysteresis in $\rho_{x x}$ of the bilayer system takes place when one of the layers is at filling factor one. Second, data of (c) and (d) show that each individual layer exhibits hysteresis when the other layer is at filling factor one, i.e., $\rho_{x x}$ of the top layer exhibits hysteresis when $\nu_{B}=1$, and vise versa.

The observation of hysteretic magneto-resistance in unbalanced bilayer systems has precedence. Zhu et al. [13] reported hysteresis in $2 \mathrm{D}$ electron systems with a parallel conducting layer. In their case, the parallel layer was a parasitic, low-mobility, doping layer at a distance of 75 to $95 \mathrm{~nm}$ away from the high-mobility 2D electrons. Similar to our data, they observed hysteresis in $\rho_{x x}$ when the layer containing the high-mobility $2 \mathrm{D}$ electrons was in a $\mathrm{QH}$ state. They also presented a simple model to explain the observed hysteresis. In their model, the hysteresis comes about because of a non-equilibrium charge distribution in the layers. As $B$ is swept, thanks to the Landau quantization, the Fermi levels of both layers oscillate. These oscillations lead to temporary imbalances between the chemical potentials of the two layers. The potential imbalance is particularly abrupt and large when the high-mobility 2D electrons enter a $\mathrm{QH}$ state as their Fermi level jumps by a significant amount, equal to the separation between the adjacent LLs. With increasing time, of course, the Fermi levels of the two layers have to come to equilibrium since the latter are shorted together via the ohmic contacts. But this equilibration can 

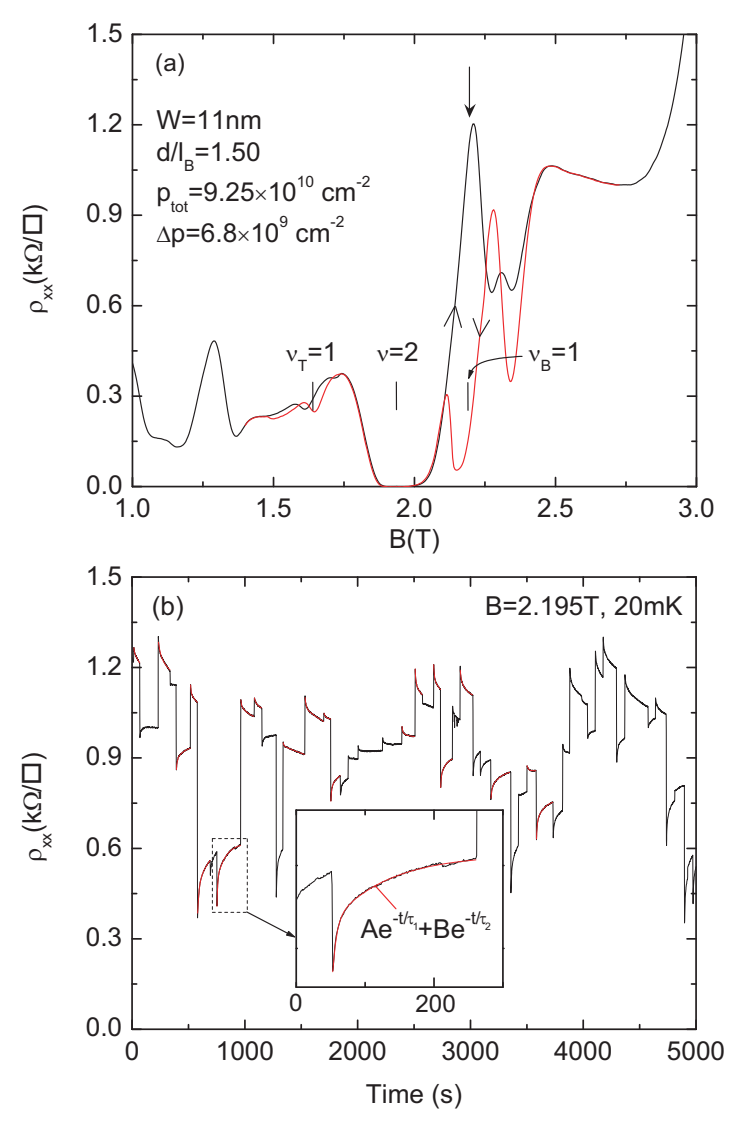

FIG. 2: (a) $\rho_{x x}$ vs. $B$ traces for another sample with $W=11 \mathrm{~nm}$, with $p_{\text {tot }}$ and $\Delta p$ as indicated. (b) $\rho_{x x}$ vs. time measured following an up-sweep of $B$ and stopping $B$ at 2.195T. $\rho_{x x}$ displays sudden jumps followed by a slow relaxation. The red lines represent double exponential fits to the slow relaxation component of the data, with time constants $\tau_{1} \sim 5 s$ and $\tau_{2} \sim 90 s$.

take a long time in the $\mathrm{QH}$ effect regime: it has to take place via the layers' edges and the ohmic contacts since the (bulk) states in the center of the 2D layer that is in the QH state are localized and the layer sheet conductivity is very small. As a result, $\rho_{x x}$, which is recorded as $B$ is swept at a finite rate, can show a hysteretic behavior. Consistent with their model, Zhu et al. found that when the $B$-sweep is interrupted in the hysteretic region and $\rho_{x x}$ is monitored as a function of time, it decays approximately exponentially toward an equilibrium value. Moreover, they found the time constant of the decay in reasonable agreement with estimates based on the parameters of their experiment.

The hysteresis in our samples resembles what Zhu et al. observe and likely has a similar origin. Our data of Fig. 1 in fact explicitly show that the hysteresis happens when one of the layers is in a $\mathrm{QH}$ state, and it is the resistivity of the layer which is not in the $\mathrm{QH}$ state that is hysteretic. The interpretation that the hysteresis indicates a charge transfer between the two layers is also
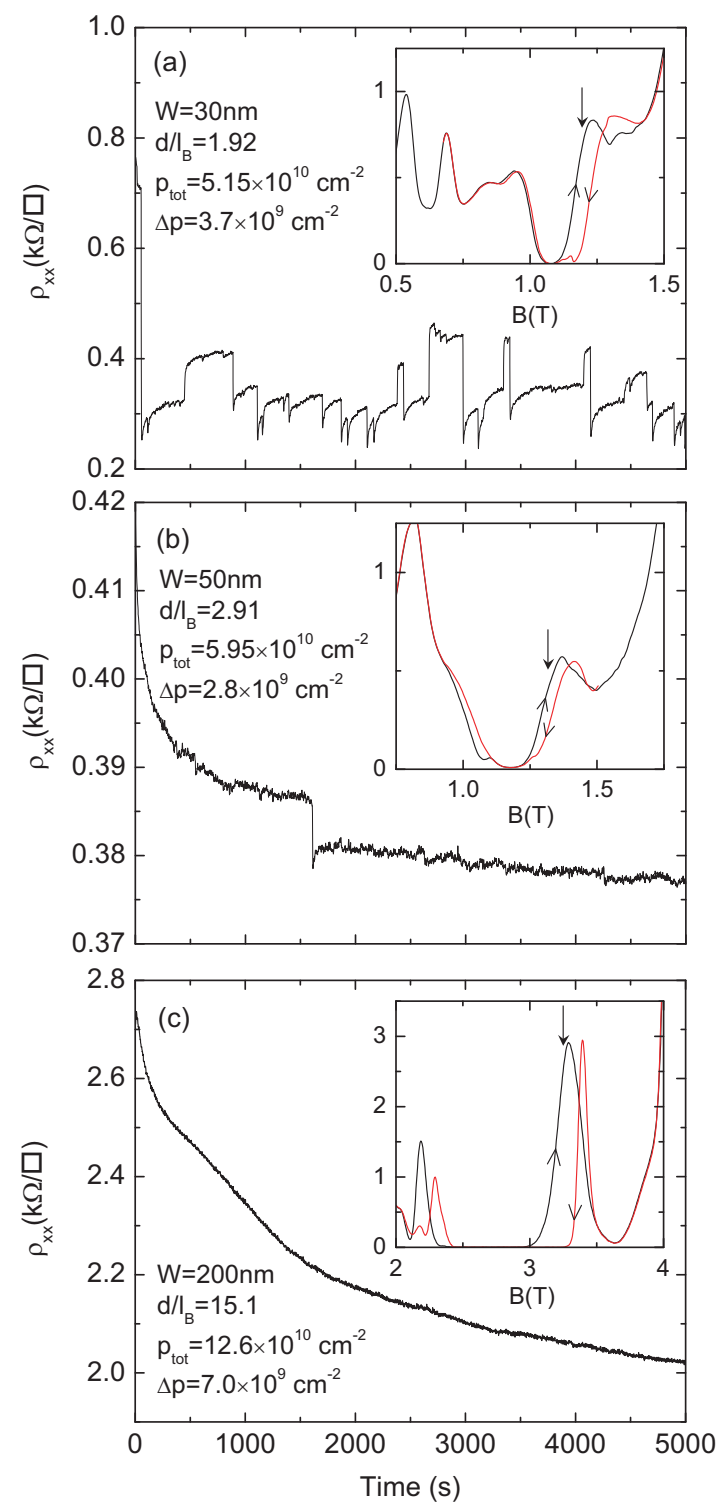

FIG. 3: Time evolution of $\rho_{x x}$ in the hysteretic region for samples with different $W, p_{t o t}$, and $\Delta p$, as indicated. The insets show $\rho_{x x}$ vs. $B$ for up- and down-sweeps. The arrow in each inset indicates the position at which the time evolution was recorded after an up-sweep of $B$.

supported by our data: the hysteresis observed in $\rho_{x x}$ of either the top or the bottom layer measured separately appears much like a horizontal shift of the trace in $B$, as if the layer had slightly different density when ramping $B$ up or down [see, e.g., Fig. 1(c)]. The time evolution of $\rho_{x x}$ in our samples, however, is qualitatively different from the observations of Zhu et al., and points to a very unusual relaxation. In the remainder of the paper, we describe this evolution and speculate on its possible origin.

We have studied a number of samples with varying $p_{t o t}$, $\Delta p$, and barrier width $7.5 \leq W \leq 200 \mathrm{~nm}$. Samples with $W=7.5 \mathrm{~nm}$ do not show any hysteresis; this is likely 
because the interlayer tunneling is sufficiently large so that the two layers stay in equilibrium during the $B$ sweep. We observe hysteresis for all samples with $11 \leq$ $W \leq 200 \mathrm{~nm}$, but the time evolution of $\rho_{x x}$ critically depends on $W$. Examples are shown in Figs. 2 and 3. For samples with $W=11 \mathrm{~nm}$ (Fig. 2), the time evolution is simply wild! It displays sudden jumps in $\rho_{x x}$, followed by a slow relaxation after each jump. Note that $\rho_{x x}$ jumps toward both higher and lower values, reminiscent of a bistability, although the jumps do not happen between fixed values of $\rho_{x x}$. In between jumps, $\rho_{x x}$ follows a slow relaxation, in the opposite direction of the jump, that can be fitted well by a double exponential [see Fig. 2(b) inset]. It is noteworthy that even when measured over days (up to $2.5 \times 10^{5} \mathrm{~s}$ ), we did not observe any tendency towards a settling of the jumps. We wish to emphasize that, outside the hysteretic region, $\rho_{x x}$ is independent of time to within less than $0.3 \%$.

We have attempted to quantify the characteristics of this evolution by two parameters: average frequency and amplitude of the jumps. Our $T$-dependence measurements show that, at a fixed $B$, the average jump amplitude decreases as $T$ increases. Not surprisingly, the jumps are no longer visible above the temperature where the hysteresis vanishes. On the other hand, the average frequency appears to be independent of $T$. Also, the jump frequency and amplitude are independent of the magnitude of the sample current, as long as the current is kept sufficiently small ( $\leq 10 \mathrm{nA})$ [14]. Interestingly, the jumps and decays appear to continue even when the current is completely turned off [15].

The sample with $W=30 \mathrm{~nm}$ shows a behavior qualitatively similar to the one with $W=11 \mathrm{~nm}$, although both the frequency and size of $\rho_{x x}$ jumps are smaller [Fig. 3(a)]. Data for the $W=50 \mathrm{~nm}$ sample, however, are qualitatively different $[($ Fig. $3(\mathrm{~b})]$ : there are by far fewer $\rho_{x x}$ jumps (typically one jump every few $10^{3} \mathrm{~s}$ ), and $\rho_{x x}$ appears to decay with time. Finally, for the $W=200 \mathrm{~nm}$ sample [Fig. 3(c)], we typically observe a simple decay with time [16]. Interestingly, for the samples of Figs. 3(b) and (c), $\rho_{x x}$ continues to decay with an ever increasing time constant.

The time evolutions we observe for the sample with $W=200 \mathrm{~nm}$ barrier [Figs. 3(c)] is qualitatively similar to the observation of Zhu et al. [13]. The time dependences for the samples with barrier widths $W=11$ and $30 \mathrm{~nm}$, however, are very unusual and cannot be understood in a simple model where the bilayer system slowly and steadily relaxes to an equilibrium state. In these samples, $\rho_{x x}$ displays sudden jumps that do not have any tendency to settle, at least over a time scale of days. We do not know the origin of these time evolutions. The sudden jumps in $\rho_{x x}$ in our bilayers with small $W$ bear some resemblance to the so-called Barkhausen jumps, which are observed in magnetic materials [1]. The Barkhausen jumps occur when the magnetic system finds a lower energy state available and one or several domains change orientation. A tantalizing speculation is that in the bilayer systems with close layer separation, the interlayer interaction acts as an opposing force to the charge transfer caused by the Fermi level difference [17]. In this scenario, the two opposing mechanisms may mediate the creation of a complicated layer charge density pattern or pseudospin domains.

Another possibility is that the observed jumps are not intrinsic to the sample, but rather triggered by external sources (e.g. electromagnetic noise). If so, it is a puzzle why the jumps are much more frequent in the bilayer samples with smaller layer separation and are seen only in the hysteretic region.

In summary we report an unusual time dependence associated with hysteretic magneto-resistance in GaAs bilayer holes with close layer separation. The resisitivity exhibits sudden jumps with time, possibly caused by a layer charge instability.

We thank DOE and NSF for support and D. Haldane for helpful discussions.
[1] G. Berttoti, Hysteresis and Magnetism (Academic Press, New York, 1998).

[2] H. Cho et al., Phys. Rev. Lett. 81, 2522 (1998).

[3] S. Kronmuller et al., Phys. Rev. Lett. bf 82, 4070 (1999).

[4] V. Piazza et al., Nature 402, 638 (1999).

[5] J. Eom et al., Science 289, 2320 (2000).

[6] E.P. De Poortere et al., Science 290, 1546 (2000).

[7] J.H. Smet et al., Phys. Rev. Lett. 86, 2412 (2001).

[8] T. Jungwirth and A.H. MacDonald, Phys. Rev. B 63, 035305 (2001).

[9] The carrier mass in GaAs 2D holes is $0.38 m_{e}$, while that of GaAs 2D electrons is $0.067 m_{e} ; m_{e}$ is the electron mass.

[10] A simple band calculation yields a tunneling energy of less than $1 \mu \mathrm{K}$ for the bilayer system studied here.

[11] J.P. Eisenstein et al., Appl. Phys. Lett. 57, 2324 (1990).

[12] The total density is kept constant to within $3 \%$. The charge transfer $\Delta p$ is measured with an accuracy of $6 \%$.
[13] J. Zhu et al., Phys. Rev. B 61, R13361 (2000).

[14] At higher currents the average amplitude of the jumps gradually decreases, likely because of Joule heating of the carriers.

[15] For example, for the sample in Fig. 2(b), after a temporary turn-off of the current (or grounding of all contacts), $\rho_{x x}$ does not recover to its value before the turn-off. If the off period is short ( $\leq 1$ minute), $\rho_{x x}$ typically follows the same decay as before the current was turned off. For long off periods, $\rho_{x x}$ attains a value that is much different than before, suggesting the occurrence of a jump even when the current was turned off.

[16] Even the $W=200 \mathrm{~nm}$ sample shows $\rho_{x x}$ jumps, but these are extremely rare. We measured four traces like in Fig. $3(\mathrm{c})$, taken over a total period of $5 \times 10^{4} \mathrm{~s}$. In two traces we observed a single jump, followed by a decay.

[17] As a measure of interalyer interaction, in Figs. 2 and 3, 
we have included values for $d / l_{B}$, the ratio between the interalyer distance $(d)$ and the magnetic lenght $\left(l_{B}\right)$. 